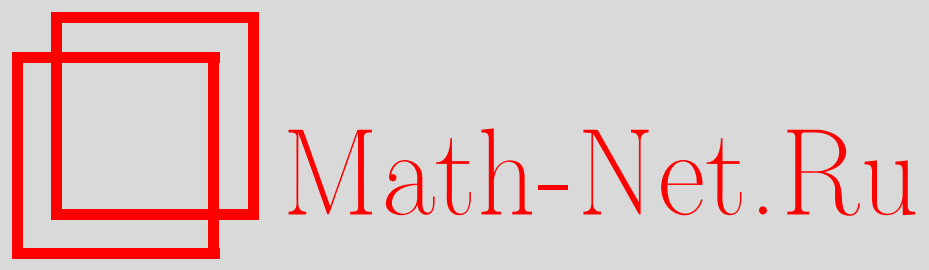

И. А. Икромов, Ф. Шарипов, О дискретном спектре неаналитической матричнозначной модели Фридрихса, Функи. анализ и его прил., 1998, том 32 , выпуск 1, 63-65

DOI: https://doi.org/10.4213/faa399

Использование Общероссийского математического портала MathNet.Ru подразумевает, что вы прочитали и согласны с пользовательским соглашением

http://www . mathnet.ru/rus/agreement

Параметры загрузки:

IP : 54.197 .217 .227

26 апреля 2023 г., $12: 26: 16$

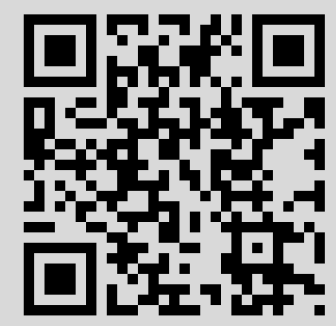


УДК 517.984 .46

\title{
О дискретном спектре неаналитической матричнозначной модели Фридрихса
}

\author{
(c) 1998. И. А. ИкРомов, Ф. ШАРИПов
}

1. Рассмотрим самосопряженный оператор $H$, действующий в гильбертовом пространстве $L^{2}\left(\mathbb{T}^{\nu}, \mathbb{C}^{n}\right)$ по формуле

$$
(H f)(x)=(H(K) f)(x)=U(x) f(x)+\int_{\mathbb{T}^{\nu}} K(x, y) f(y) d y .
$$

Здесь $\mathbb{T}^{\nu}$ - это $\nu$-мерный тор, $\mathbb{C}^{n}$ есть $n$-мерное комплексное пространство, $L^{2}\left(\mathbb{T}^{\nu}, \mathbb{C}^{n}\right)$ - комплексное гильбертово пространство квадратично суммируемых по норме функций на $\mathbb{T}^{\nu}$ со значениями в $\mathbb{C}^{n}$, а $U(x)$ и $K(x, y)-$ непрерывные функции соответственно на $\mathbb{T}^{\nu}$ и $\mathbb{T}^{\nu} \times \mathbb{T}^{\nu}$ со значениями в пространстве $M_{n}(\mathbb{C})$ комплексных $n \times n$-матриц, удовлетворяющие условиям

$$
U^{*}(x)=U(x), \quad K^{*}(x, y)=K(y, x),
$$

где * означает переход к сопряженной матрице.

Впервые оператор вида $(1)$ в пространстве $L^{2}(-1,1)$ был рассмотрен К. Фридрихсом $[1,2]$ в случае $U(x)=x$ в качестве простой модели теории возмушений непрерывного спектра. Непосредственное развитие модель Фридрихса получила в работах [3-9] и др.

В случае когда матричнозначные функции $U(x)$ и $K(x, y)$ являются аналитическими, спектральные свойства оператора вида (1) изучены в работах $[6,7,9-11]$. В частности, при некоторых дополнительных условиях доказана конечность дискретного спектра оператора $H$.

В этой работе установлена конечность дискретного спектра оператора $H$ для более широкого класса функций $U(x)$ и $K(x, y)$.

2. Обозначим через $\Sigma_{\text {ess }}(H)$ существенный спектр оператора $H$, а через $\Delta_{z}(x)$ определитель матрицы $U(x)-z E$, где $E-$ единичная матрица, $z \in \mathbb{C}$. Из теоремы Вейля о существенном спектре и принципа минимакса (см. [12]) следует справедливость следующих соотношений:

$$
\Sigma_{\text {ess }}(H)=\bigcup_{x \in \mathbb{T}^{\nu}}\left\{z \in \mathbb{C}: \Delta_{z}(x)=0\right\}=\bigcup_{i=1}^{k}\left[m_{i}, M_{i}\right],
$$

где $\left[m_{i}, M_{i}\right](i=1, \ldots, k, k \leqslant n)$ - попарно непересекающиеся сегменты.

Обозначим через $\Gamma$ множество $\left\{m_{1}, \ldots, m_{k}, M_{1}, \ldots, M_{k}\right\}$.

Лемма 1. Для любого фиксированного $z \in \Gamma$ число $A=0$ является глобальным экстремальным значением непрерывной на торе $\mathbb{T}^{\nu}$ вещественнозначной функции $\Delta_{z}(x)$.

* Исследование было частично поддержано Узбекским фондом фундаментальных исследований (конт. № 44) и Международным научным фондом (грант No. MGM000). 
ОПРЕДЕЛЕНИЕ 1 . Пусть $\varphi(x)$ - непрерывная вещественнозначная функция на торе $\mathbb{T}^{\nu}$. Экстремальная точка $x^{0} \in \mathbb{T}^{\nu}$ функции $\varphi(x)$ называется конечнократной, если существуют числа $m>0, c>0$ и окрестность $V\left(x^{0}\right)$ точки $x^{0}$, такие, что для каждого $x \in V\left(x^{0}\right)$ справедливо неравенство

$$
\left|\varphi(x)-\varphi\left(x^{0}\right)\right| \geqslant c\left|x-x^{0}\right|^{m}, \quad \text { где }\left|x-x^{0}\right|^{2}=\left(x_{1}-x_{1}^{0}\right)^{2}+\cdots+\left(x_{\nu}-x_{\nu}^{0}\right)^{2} \text {. }
$$

В противном случае экстремальная точка $x^{0}$ называется бесконечнократной. Точная нижняя грань множества чисел $m>0$, для которых выполняется условие (3), называется кратностью экстремальной точки $x^{0}$. Кратностью экстремального значения $A$ функции $\varphi(x)$ называется сумма кратностей всех экстремальных точек из $\varphi^{-1}(A)$, где $\varphi^{-1}(A)$ - полный прообраз точки $A$ при отображении $\varphi$.

Обозначим через $C^{\alpha+0}\left(\mathbb{T}^{\nu} \times \mathbb{T}^{\nu}, M_{n}(\mathbb{C})\right)$ пространство непрерывных матричнозначных функций $K(x, y)$ на $\mathbb{T}^{\nu} \times \mathbb{T}^{\nu}$, таких, что для любого мультииндекса $\beta$ с $|\beta| \leqslant[\alpha]$ производная $K^{(\beta)}(x, y)$ удовлетворяет условию Гёльдера с показателем $\{\alpha\}+\varepsilon$ для некоторого $\varepsilon>0$, где $[\alpha]$ - целая часть числа $\alpha$ и $\{\alpha\}=\alpha-[\alpha]$.

ТЕОРема 1. Предположим, что для каждого фиксированного $z \in \Gamma$ число $A=0$ является экстремальным значением функции $\Delta_{z}(x)$ кратности $\leqslant \mu$, где $\mu$ - некоторое положительное число. Тогда для любой функиии $K(x, y)$ из класса $C^{2 \mu-\nu / 2+0}\left(\mathbb{T}^{\nu} \times \mathbb{T}^{\nu}, M_{n}(\mathbb{C})\right)$ оператор $H$ имеет лишь конечное число собственных значений (с учетом кратностей) вне существенного спектра.

При доказательстве теоремы 1 используется следующая лемма:

ЛЕмма 2. Пусть $z \in \Gamma$. Если матричнозначная функиия $K(x, y) / \Delta_{z}(x)$

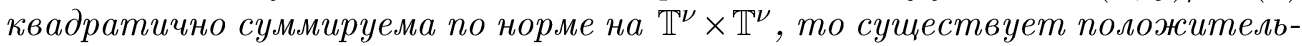
ное число $\varepsilon=\varepsilon(z)$, такое, что оператор $H$, определяемьй по формуле (1), имеет лишь конечное число собственньх значений (с учетом кратностей) в множестве $(z-\varepsilon, z+\varepsilon) \backslash \Sigma_{\mathrm{ess}}(H)$.

СХЕМА ДОКАЗАТЕЛЬСТВА ТЕОРЕМЫ 1 . Из условий теоремы 1 следует, что для каждого $z \in \Gamma$ функцию $K(x, y)$ можно представить в виде

$$
K(x, y)=K_{1}(x, y)+K_{2}(x, y)
$$

так, чтобы выполнялись условия

$$
\left\|K_{1}(x, y) / \Delta_{z}(x)\right\| \in L^{2}\left(\mathbb{T}^{\nu} \times \mathbb{T}^{\nu}\right), \quad K_{1}^{*}(x, y)=K_{1}(y, x), \quad K_{2}^{*}(x, y)=K_{2}(y, x)
$$

и интегральный оператор с ядром $K_{2}(x, y)$ в пространстве $L^{2}\left(\mathbb{T}^{\nu}, \mathbb{C}^{n}\right)$ являлся конечномерным. В силу леммы 2 существует положительное число $\varepsilon=\varepsilon(z)$, такое, что оператор $H\left(K_{1}\right)$ имеет лишь конечное число собственных значений (с учетом кратностей) в множестве $(z-\varepsilon, z+\varepsilon) \backslash \Sigma_{\mathrm{ess}}(H)$. Так как при конечномерном возмущении мошность дискретного спектра меняется лишь на конечное число (см. [12]), то оператор $H(K)=H\left(K_{1}+K_{2}\right)$ также имеет конечное число собственных значений в множестве $(z-\varepsilon, z+\varepsilon) \backslash \Sigma_{\text {ess }}(H)$. Поскольку точками сгущения дискретного спектра оператора $H$ могут быть только точки из $Г$, то отсюда следует справедливость утверждения теоремы 1. 
3. Предположим, что матричнозначная функция $U(x)$ является аналитической. Тогда для каждого фиксированного $z \in \mathbb{R}$ функция $\Delta_{z}(x)$ является вещественно-аналитической на $\mathbb{T}^{\nu}$. Из неравенства Лоясеевича (см. [13]) следует, что изолированная экстремальная точка вещественно-аналитической функции на торе $\mathbb{T}^{\nu}$ является конечнократной экстремальной точкой в смысле определения 1. Поэтому из теоремы 1 следует справедливость следующей теоремы, которая является обобщением аналогичных теорем из работ $[6,9]$ о конечности дискретного спектра.

ТЕОРема 2. Пусть матричнозначная функиия $U(x)$ аналитична на $\mathbb{T}^{\nu} u$ для каждого $z \in \Gamma$ множество $\Delta_{z}^{-1}(0)$ конечно. Тогда существует положительное число $s>0$, такое, что для каждой матричнозначной функиии $K(x, y)$ из класса $C^{s+0}\left(\mathbb{T}^{\nu} \times \mathbb{T}^{\nu}, M_{n}(\mathbb{C})\right)$ оператор $H$ имеет конечное число собственных значений ( с учетом кратностей) вне существенного спектра.

Как показывают примеры, в теореме 2 условие конечности множества $\Delta_{z}^{-1}(0)$ является существенным.

4. Пусть $\varphi \in C^{s}\left(\mathbb{T}^{\nu}\right)$, где $s$ - натуральное число. Через $J_{a}^{s} \varphi, a \in \mathbb{T}^{\nu}$, обозначается $s$-струя функции $\varphi$ в точке $a$ (см. [13]). Из теоремы 1 вытекает следующая

Tеорема 3. Пусть $U(x) \in C^{\mu+3}\left(\mathbb{T}^{\nu}, M_{n}(\mathbb{C})\right)$, где $\mu$ - некоторое натуральное число. Если для любых $z \in \Gamma u a \in \Delta_{z}^{-1}(0)=L$ существует гладкая Функиия $\psi(x)$ на $\mathbb{T}^{\nu}$, для которой точка а является критической точкой кратности $n(a)$ u $J_{a}^{\mu+1} \Delta_{z}=J_{a}^{\mu+1} \psi, \sum_{a \in L} n(a) \leqslant \mu$, то для любой функиии $K(x, y) \in C^{2 \mu+2-\nu / 2+0}\left(\mathbb{T}^{\nu} \times \mathbb{T}^{\nu}, M_{n}(\mathbb{C})\right)$ оператор $H$ имеет конечное число собственных значений ( с учетом кратностей) вне существенного спектра.

Авторы выражают глубокую благодарность С. Н. Лакаеву за полезные обсуждения, а также Р. А. Минлосу и рецензентам за полезные замечания.

\section{ЛитеРАтУРА}

1. Friedrichs K. Ann. Math., 97, 247-274 (1938). 2. Friedrichs K. Comm. Pure Appl. Math., 1, 361-406 (1948). 3. Ладыженская О. А., Фаддеев Л. Д. Докл. АН СССР, 145, № 2, 301-304 (1962). 4. Фаддеев Л. Д. Труды МИАН СССР, 73, 292-313 (1964). 5. Павлов Б. С., Петрас С. В. Функц. анализ и его прил., 4, вып. 2, 54-56 (1970). 6. Lakaev S. N. Comm. Math. Univ. Carolinae, 27, 341-357 (1986). 7. Лакаев С. Н. Докл. АН УзССР, № 4, 9-10 (1979). 8. Дьнкин Е. М., Набоко С. Н., Яковлев С. И. Алгебра и анализ, 3, вып. 2, 77-90 (1991). 9. Abdullaev Zh. I., Lakaev S. N. Advances in Soviet Math., 5, 1-37 (1991). 10. Minlos R. A., Mogilner A. I. Schrödinger Operators, Standard and Nonstandard, P. Exner, P. Seba, eds., Inst. Nucl. Res., Dubna (USSR), Singapore, 1988, p. 243-257. 11. Маматов Ш. С., Минлос Р. А. Теор. матем. физ., 79, 163-180 (1989). 12. Рид М., Саймон Б. Методы современной математической физики, т. 4, Анализ операторов. Мир, М., 1982. 13. Мальгранж Б. Идеалы дифференцируемых функций. Мир, М., 1968.

Самаркандский государственный университет, механико-математический факультет 